\title{
Contributions to thermal constraints management in radial active distribution systems
}

\author{
Ilya Bilibin, Florin Capitanescu* \\ Interdisciplinary Centre for Security, Reliability and Trust (SnT), University of \\ Luxembourg, 4 rue Alphonse Weicker, L-2721 Luxembourg, Tel. +352 466644 5345, Fax: \\ +3524666445669.
}

\begin{abstract}
This paper deals with centralized thermal overload management in active radial distribution systems that host a significant amount of distributed generation (DG). We investigate the benefits of using remotely controlled switches to reduce the amount of curtailed DG to remove overload. To this end we extend an existing optimization model to the problem of minimizing the nonfirm DG curtailment to remove overload. We discuss the pros and cons of the various overload management goals given the particular features of radial distribution grids and propose, wherever possible, the use of a power flow tracing-based procedure to select the non-firm generators that should participate in overload removal. Although the approach focuses on overload removal it also inhibits violation of operational constraints such as voltage limits that may occur due to network reconfiguration. We prove the interest and feasibility of our approach in four distribution networks.
\end{abstract}

Keywords: Active distribution system, congestion management,

\footnotetext{
*Corresponding author

Email addresses: ilya.bilibin@uni.lu (Ilya Bilibin), fcapitanescu@yahoo.com (Florin Capitanescu)
} 
distributed generation, network switching, network reconfiguration, optimal power flow, smart grid

\section{Introduction}

In order to meet the more stringent environmental constraints, many distribution systems (DSs) host increasing amounts of distributed generation (DG) (e.g. wind, photovoltaic, etc.) [1, 2]. This may lead to a significant increase in reverse power flows and thereby to thermal and/or voltage constraints among other operational issues. Medium voltage distribution systems are generally either (and mostly) voltage constrained or thermally constrained. Voltage (raise) constraints generally arise in very long rural networks, whereas thermal constraints $[3,4,5]$ in lines/cables/transformers may prevail in networks with short lines or with relatively large nominal voltage (e.g. $20 \mathrm{kV}$ to $33 \mathrm{kV}$ ).

There are two philosophies for determining the allowed DG penetration level in a DS [1]: passive DSs and active DSs. The former paradigm is based on the "fit-and-forget" principle (i.e. a new DG is accommodated only if this does not lead to operational constraints violation under worst operating scenario). This approach is very conservative and may prevent achieving the required green energy target and harvesting DG benefits (e.g. reduction of: investments in new assets, losses, load peaks, etc.). Active DS concept is a way to significantly increase DG penetration by managing DG output and other control means through centralized [6, 4, 5] or distributed [7] control schemes.

In this work we focus on thermally constrained active DSs in which we 
assume a centralized management of thermal constraints.

Several approaches have been devoted to the overload management in active DSs such as: (time-series) optimal power flow (OPF) [6, 4], constraint programming [5], sensitivity-based [7], etc. OPF [8] is an essential tool to manage constraints in both transmission [9] and distribution systems [6, 4]. In DSs it provides optimal DG curtailment to remove constraints according to a given goal (e.g. minimizing either the MW curtailed or the curtailment cost [10]) or DG connection agreements (e.g. last-in, first-off [4]). However, these approaches do not consider network switching as an option.

The main contribution of this work is to investigate the benefits of relying on remotely controlled switches to reduce the DG curtailment. This leads to pose a mixed integer nonlinear programming (MINLP) problem. To reduce the computational burden of MINLP problem [12], the latter can be reformulated, for radial DSs, as a more tractable equivalent mixed integer quadratically constrained (MIQC) problem, as demonstrated in [13] for power losses minimization by means of network reconfiguration. In this work we further extend the model in [13] to the problem of overload management and extend significantly our previous approach [14]. Another contributions of the paper are: an analysis of the pros and cons of constraint management goals, and a power flow tracing scheme to select only the DG units that are truly responsible for overload as candidate for curtailment in OPF.

The rest of the paper is organized as follows. Section 2 discusses the main features of the overload management scheme. Section 3 presents the mathematical model of the optimization approach. Section 4 provides numerical results with the method and section 5 concludes. 


\section{Features of the proposed approach}

\subsection{Regulatory framework}

In most distribution systems (e.g. in Europe), in order to ensure fair access and competition between DG units, electricity market and distribution system operation are unbundled; hence the distribution system operator (DSO) cannot own DG [10]. The DG access to the grid relies on DG connection agreements [10] (e.g. "last-in, first-off" principle in U.K. [4]). In this framework, according to the DG connection agreement, one can distinguish between "firm" DG units (generators that cannot be curtailed to remove grid constraints as they invested in grid reinforcement; these DG units are accommodated based on a worst-case scenario) and "non-firm" DG units (generators that accepted to be occasionally curtailed as overload occurs because the lost revenue is deemed more advantageous economically than grid reinforcement option) [4]. As this unbundling may lead to poor operation performances of the DS and or limited DG penetration level, DSO-owned DG frameworks are advocated [16]. Furthermore, regulation may also differ in whether the owners of curtailed non-firm DG units receive a compensation to cover the lost revenue (if this is the case then the DSO looks for minimizing the payments towards the owners of curtailed DG units [10]; otherwise the DSO seeks to minmize the overall DG curtailed energy).

We conclude that most regulatory frameworks differ basically in two respects: the choice of non-firm DG units participating in curtailment and the optimization goal. Bearing this in mind we devise in Section 3 an optimization approach for overload management which is versatile enough to be applicable in various regulatory frameworks (e.g. by properly choosing 


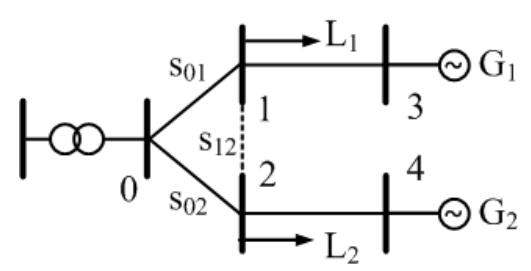

Figure 1: Illustrative 5-bus distribution network

the objective function, some variables and some constraints), including both non-dispatchable and dispatchable DG [15].

\subsection{Illustrating the benefits of remotely controlled switches option}

The benefits of remotely switching in terms of curtailed energy saving are briefly illustrated by running our optimizer for 24-hours generation/load patterns on the 5-bus system shown in Fig. 1 (the data set of this test case are provided in the Appendix). Sectionalizing switches $s_{01}$ and $s_{02}$ and tie switch $s_{12}$ are remotely controlled. G1 is a photovoltaic unit and G2 is a wind unit. The thermal limits of lines $0-1$ and $0-2$ prevent larger power injections into the higher voltage grid and hence hosting larger amounts of DG.

Fig. 2 shows the unconstrained generators profiles (with dotted line), the constrained generators profiles with only DG curtailment as control option (with dashed line), and the constrained generators profiles with remotely controlled switches as additional control option (with continuous line). The grey areas represent the energy saved thanks to switching actions, provided in Table 1, clearly highlighting the value of this control means. These gains are obtained by redirecting the output of G2 through lines 1-2 and 0-1 when the load L1 is high and generation G1 is small as well as by redirecting 


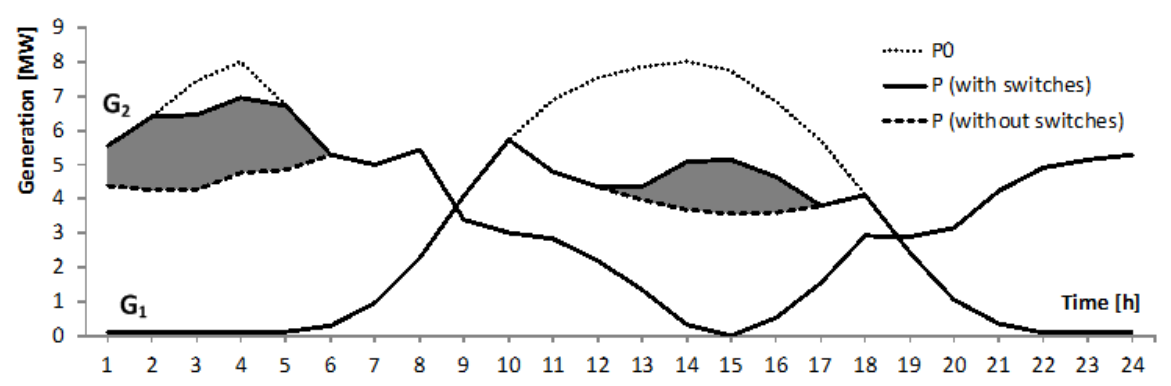

Figure 2: Energy savings thanks to switching actions

Table 1: Hourly status of remotely controlled switches

\begin{tabular}{|c|c|c|c|c|c|c|c|c|c|c|c|c|c|c|c|c|c|c|c|c|c|c|c|c|}
\hline switch & 1 & 2 & 3 & & 5 & 6 & 7 & 8 & 9 & 10 & 11 & 12 & 13 & 14 & 15 & 16 & 17 & 18 & 19 & 20 & 21 & 22 & 23 & 24 \\
\hline$s_{01}$ & 0 & 0 & 0 & 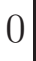 & 0 & 1 & 1 & 1 & 1 & 1 & 1 & 1 & 1 & 1 & 1 & 1 & 1 & 1 & 1 & 1 & 1 & 1 & 1 & 1 \\
\hline$s_{02}$ & 1 & 1 & 1 & 1 & 1 & 1 & 1 & 1 & 1 & 1 & 1 & 1 & 0 & 0 & 0 & 0 & 1 & 1 & 1 & 1 & 1 & 1 & 1 & 1 \\
\hline$s_{12}$ & 1 & 1 & 1 & & & 0 & 0 & 0 & 0 & 0 & 0 & 0 & 1 & 1 & 1 & 1 & 0 & 0 & 0 & 0 & 0 & 0 & 0 & 0 \\
\hline
\end{tabular}

the output of G1 through lines 1-2 and 0-2 when the load L2 is high and generation G2 is small. Switches status change occur at hours 6, 13, and 17 .

\subsection{Analysis of possible choices for the objective function}

From the perspective of maximizing the amount of non-firm DG accommodated in the DS (or equivalently minimizing their curtailment) and offering incentives for a fair competition regarding the connection access of DG units to the grid, we assess the pros and cons of three objectives: the norm $L_{1}(1)$, a weighted linear objective $L_{1 w}(2)$, and the norm $L_{2}(3)$ :

$$
\begin{gathered}
L_{1}=\min \sum_{i \in G}\left(P_{g i}^{0}-P_{g i}\right), \\
L_{1 w}=\min \sum_{i \in G} w_{g i}\left(P_{g i}^{0}-P_{g i}\right),
\end{gathered}
$$




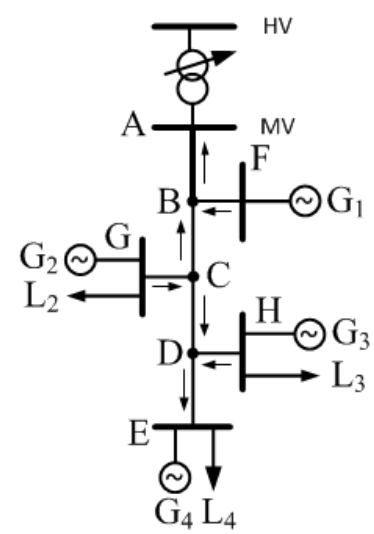

Figure 3: Simple distribution grid

$$
L_{2}=\min \sum_{i \in G}\left(P_{g i}^{0}-P_{g i}\right)^{2}
$$

where, for the non-firm unit $i, P_{g i}^{0}$ is its power produced at a given time and the weight $w_{g i}$ stands for either its curtailment cost, or is inversely proportional to its impact on the overload.

We illustrate this comparison using the simple distribution system in Fig. 3 (the data set of this test case are provided in the Appendix), and assuming that G1, G2, G3, and G4 are non-firm wind power generators. In this figure the arrows indicate the direction of active power flows.

Let us assume that this grid operates in a worst-case scenario (i.e. minimum load vs. maximum feed-in) and that line AB becomes overloaded due to a significant feed-in power of DG.

Table 2 provides, the minimum overall MW curtailment of DG units provided by the three objectives and for two approaches to select DG participating to overload removal (all generators participate versus some generators selected by the proposed approach described in next sub-section). 
Table 2: Non-firm DG units curtailment (MW)

\begin{tabular}{|c|c|c|c|c|c|}
\hline gen & $\begin{array}{c}L_{1} \\
\text { all }\end{array}$ & $\begin{array}{c}L_{2} \\
\text { all }\end{array}$ & $\begin{array}{c}L_{1} \\
\text { selected }\end{array}$ & $\begin{array}{c}L_{2} \\
\text { selected }\end{array}$ & $\begin{array}{c}L_{1 w} \\
\text { selected }\end{array}$ \\
\hline G1 & 0.00 & -0.142 & -0.565 & -0.283 & -0.459 \\
\hline G2 & 0.00 & -0.141 & 0.00 & -0.283 & -0.107 \\
\hline G3 & 0.00 & -0.141 & - & - & - \\
\hline G4 & -0.562 & -0.141 & - & - & - \\
\hline total & 0.562 & 0.567 & 0.565 & 0.566 & 0.566 \\
\hline
\end{tabular}

It is important to note that due to the tree structure of radial distribution systems all generators downstream the overload are practically equally effective to remove an overload in the main feeder. This assertion is confirmed by the quadratic $L_{2}$ norm objective (see column labeled " $L_{2}$ all") as generators G1, G2, G3, and G4 share practically equally the effort of overload removal, and the overall amount of curtailment is only slightly higher (due to losses) than the $L_{1}$ norm. This objective may be of interest especially in a context of active DSs where the grid is insufficiently observable to allow tracing precisely the flow of electricity.

As expected the norm $L_{1}$ leads to the least overall curtailment as it calls for curtailment of the most effective DG units to remove overload (see column labeled " $L_{1}$ all"). This objective makes sense in a context where the DSO is also the owner of all DG units on the DS [15]. However, it may lead to discriminatory DG access in a context with independent DG power producers, as it focuses the entire effort on the most sensitive generator G4, regardless the fact that all four generators are practically equally effective. 
For an active DSs reasonably observable and future smart grids, a powertracing criterion allows identifying the DG units truly responsible of overload and favors the objective $L_{1 w}(2)$, which will fairly penalize only the responsible units. In this example, both responsible generators G1, G2 are curtailed based on their relative weights $w_{g i}$, computed according to the active power flow injected in the overloaded line i.e. 3.0 MW and 0.7 MW, respectively.

We conclude that although slightly less efficient in terms of overall curtailment compared to $L_{1}$ norm, objectives $L_{2}$ and $L_{1 w}$, with generators selected appropriately, offer incentives for a fair connection access of DG to the grid.

\subsection{Selection of non-firm DG candidate for curtailment}

We propose that the generation balanced locally should be stimulated (as it decreases losses in both transmission and distribution systems) and hence not participate in overload removal. For instance, generator G4 must not be curtailed as it produces less power than its local load L4. Furthermore, we propose that only generators for which the active power flow has the same sense from the point of connection as far as upstream the overload should participate in optimization. Therefore, although generator G3 produces more power than its local load L3, it should not participate in overload removal as its surplus of power does not flow from $\mathrm{D}$ to $\mathrm{C}$ in the same sense as the overload (see Fig. 3). According to this DG selection criterion only generators G1 and G2 must be reduced as only they induce power flows in the grid steadily in the same sense as the overload (see Fig. 3).

Note that the ideas of DG selection for overload removal fit also into power flow tracing methods developed for transmission grid [17, 18]. However, unlike the meshed transmission system, where it is not always possible to 
distinguish which part of a power flow comes from which unit $[17,18]$, the electricity flows in a radial system can be distinctively traced in most cases.

Depending on the network observability, this selection criterion can be used either as a yes/no DG unit participation to curtailment with $L_{1}$ or $L_{2}$ objectives, or as a refined weight-based participation with $L_{1 w}$ objective.

\section{Formulation of the optimization approach}

Let $\mathrm{N}, \mathrm{G}, \mathrm{L}$, and $\mathrm{S}$ denote the set of respectively: nodes, DG units, all lines, and the subset of lines with remotely controlled switches/breakers.

\subsection{Objective function and the control variables}

The goal of the overload management procedure is to minimize the (cost of) active power curtailment of non-firm DG units to remove overload, according to any of the objectives (1), (2), or (3), which is suitable in a given context. However, as the problem includes remotely controlled switches, we use a composite objective which accounts for a fictitious cost of switching actions (which can be seen as maintenance cost and/or reflect the DSO preference towards minimizing the number of switching actions and/or acting only when the reduction in generation curtailment is significant):

$$
\min \sum_{i \in G} w_{g i}\left(P_{g i}^{0}-P_{g i}\right)+w_{s} \sum_{i j \in S}\left|s_{i j}-s_{i j}^{0}\right|,
$$

where $s_{i j}$ models the dual status connected/disconnected of the line switch $i j$ and $s_{i j}^{0}$ denotes its current value, and $w_{s}$ weight to be chosen by the DSO trades-off the MW curtailed and the number of switching actions (e.g. a large value of $w_{s}$ reflects DSO unwillingness to rely on switching). 
Note that depending on the regulatory framework this composite objective could be also translated into economic terms as the minimization of the DG owners reimbursement for their loss of revenue due to curtailed energy plus the wear and tear cost of switching, $w_{g i}$ and $w_{s}$ becoming actual costs.

The control variables of the problem are: the status on/off and the active/reactive powers of DG units, and the status of remotely controlled sectionalizing switches, tie switches, and breakers.

\subsection{Problem constraints}

\subsubsection{Alternative power flow model for radial distribution grids}

The remaining part of the optimization problem extends the model ${ }^{1}$ in [13] to the overload management application. The model [13] consists, for a grid with $n+1$ buses, in replacing the $2 n$ conventional nonlinear power flow equations with a set of $3 n$ equations ( $2 n$ linear and $n$ quadratic). This is done by replacing the conventional complex voltage unknowns $\left(V_{i}, \theta_{i}\right)$ with two variables per branch $\left(W_{i j}\right.$ and $\left.T_{i j}\right)$ and one per bus $\left(U_{i}\right)$ as follows:

$$
\begin{aligned}
& U_{i}=V_{i}^{2} \\
& W_{i j}=V_{i} V_{j} \cos \left(\theta_{i}-\theta_{j}\right) \\
& T_{i j}=V_{i} V_{j} \sin \left(\theta_{i}-\theta_{j}\right),
\end{aligned}
$$

where $W_{i j}=W_{j i}$ and $T_{i j}=-T_{j i}$.

\footnotetext{
${ }^{1}$ This relies in turn on the power flow model for radial distribution systems in [19].
} 
The set of power flow equations comprises $2 n$ linear equations $(i \in N)$ :

$$
\begin{aligned}
P_{g i}-P_{c i} & = & \sum_{i j} P_{i j} & =\sum_{i j} g_{i j} U_{i}-g_{i j} W_{i j}-b_{i j} T_{i j} \\
Q_{g i}-Q_{c i} & = & \sum_{i j} Q_{i j} & =\sum_{i j}-\left(b_{i j}+b_{i j}^{s h}\right) U_{i}+b_{i j} W_{i j}-g_{i j} T_{i j}
\end{aligned}
$$

together with the $n$ quadratic constraints:

$$
U_{i} U_{j}=W_{i j}^{2}+T_{i j}^{2}, i j \in L \backslash S
$$

3.2.2. Longitudinal current limit for lines with fixed switches

$$
I_{i j}^{2}=\left(g_{i j}^{2}+b_{i j}^{2}\right)\left(U_{i}+U_{j}-2 W_{i j}\right) \leq\left(I_{i j}^{\max }\right)^{2}, i j \in L \backslash S
$$

\subsubsection{Constraints modeling the status of lines with remotely controlled switches} $(i j \in S)$

To model the switching operations and consequently whether a branch is connected or disconnected we use the following set of constraints [13]:

$$
\begin{aligned}
& -s_{i j} P_{i j}^{\max } \leq P_{i j} \leq s_{i j} P_{i j}^{\max } \\
& -s_{i j} Q_{i j}^{\max } \leq Q_{i j} \leq s_{i j} Q_{i j}^{\max } \\
& I_{i j}^{2} \leq K_{I}\left(1-s_{i j}\right)+s_{i j}\left(I_{i j}^{\max }\right)^{2} \\
& -K_{e}\left(1-s_{i j}\right) \leq U_{i} U_{j}-W_{i j}^{2}-T_{i j}^{2} \leq K_{e}\left(1-s_{i j}\right) \\
& -K_{W}\left(1-s_{i j}\right) \leq W_{i j}-W_{j i} \leq K_{W}\left(1-s_{i j}\right) \\
& -K_{T}\left(1-s_{i j}\right) \leq T_{i j}+T_{j i} \leq K_{T}\left(1-s_{i j}\right),
\end{aligned}
$$

where $P_{i j}$ and $Q_{i j}$ are given by (8) and (9), the constants $K_{I}, K_{e}, K_{W}, K_{T}$ being properly chosen as explained in [13]. 
Observe that for a connected line (i.e. $s_{i j}=1$ ) constraints (12)-(13) recover original active/reactive power flow limits, (14) and (15) take the same form as (11) and (10), and (16)-(17) provides the same outcome as (6)-(7) i.e. $W_{i j}=W_{j i}$ and $T_{i j}=-T_{j i}$. Otherwise, if a line is disconnected (i.e. $s_{i j}=0$ ) it implies that $P_{i j}=P_{j i}=0, Q_{i j}=Q_{j i}=0$ while the other constraints (14)-(17) are relaxed.

\subsubsection{Voltage magnitude limits}

$$
V_{i \min }^{2} \leq U_{i} \leq V_{i \max }^{2}, i \in N
$$

3.2.5. On/off status and active/reactive powers bounds of $D G$

$$
\begin{aligned}
& s_{g i} P_{g i}^{\min } \leq P_{g i} \leq s_{g i} P_{g i}^{0}, i \in G \\
& s_{g i} Q_{g i}^{\min } \leq Q_{g i} \leq s_{g i} Q_{g i}^{\max }, i \in G,
\end{aligned}
$$

where the binary variable $s_{g i}$ models the connection status of the generator $i\left(s_{g i}=1\right.$ if the generator is connected and $s_{g i}=0$ if it is shut down).

Note that in this approach we consider that DG units can shift their reactive power so as to reduce the overall amount of active power curtailed but other typical reactive power control modes of DG (e.g. constant power factor and in particular unity power factor) can be easily taken into account.

\subsubsection{Necessary radiality constraint}

$$
\sum_{i j \in S} s_{i j}=\sum_{i j \in S} s_{i j}^{0}
$$


which expresses the fact that the sum of statuses of lines with remote controlled switches must not change after switching actions. Because this constraint may be however insufficient to ensure radiality in some grids where there are some zero-injection nodes [13], we adopt a practical solution and replace each zero-injection bus with a very small reactive power load (of value slightly above the power flow convergence tolerance), change which practically does not affect the final result of the optimization.

\subsubsection{Constraint limiting the number of switching actions}

$$
\sum_{i j \in S}\left|s_{i j}-s_{i j}^{0}\right| \leq \Delta S_{s w}
$$

which models the DSO practical operational need that is not using more than a specified number of switching $\Delta S_{s w}$ to remove overload in real-time. This constraint can also be extended so as to model DSO preference to limit the number of switching actions over a day.

\subsection{Remarks}

Note that the overload management model (4)-(22) constitutes a very convenient and tractable MIQC problem (which is equivalent to the original MINLP), the only nonlinear (but quadratic) constraints are (10) and (15). The differences compared to the model in [13] are: objective (4), the control variables related to DG units, and constraints (19)-(22).

Unlike the (generally) off-line problem of network reconfiguration for loss minimization where all (manually and remotely controlled) switches are taken as decision variable which leads to a very large combinatorial space [13], in our procedure this combinatorial space is drastically limited due to the small 
ratio between the number of remotely controlled switches/breakers and the number of all switches.

\subsection{Prerequisite for potential implementation in a real environment}

As any centralized scheme $[3,4,5]$, the proposed optimization approach relies on the output of a state estimator. Although distribution systems are traditionally poorly observable, having measurements mainly at the substation, it has been shown that an acceptable system observability does not require a substantial number of additional measurements being deployed [20]. The state estimation computation can furthermore be enriched with pseudomeasurements stemming from load forecast (it is expected that this forecast will be significantly improved thanks to the information provided by smart meters, which are under deployment in many countries worldwide) and DG forecast. Also, the state estimation can take advantage from the fact that both load and DG output forecasts are more accurate on short-term basis.

\section{Numerical results}

\subsection{Description of the test systems}

We test the proposed approach on four distribution systems: a 33-bus $12.66 \mathrm{kV}$ benchmark distribution grid [21], a 61-bus $20 \mathrm{kV}$ modified real-world distribution network model provided by CREOS (the grid operator in Luxembourg), an 84-bus $11.4 \mathrm{kV}$ real-world distribution system in Taiwan [23], and a 136-bus $13.8 \mathrm{kV}$ real-world distribution system in Brazil [24].

In order to facilitate comparison and benchmarking for the 33-bus, 84bus, and 136-bus test systems we use the same benchmark data sets provided in [21], [23], and [24], respectively. However, to illustrate the proposed 
Table 3: Characteristics of test distribution systems

\begin{tabular}{|c|c|c|c|c|c|c|}
\hline nodes & lines & $\begin{array}{c}\text { sectionalizing } \\
\text { switches }\end{array}$ & $\begin{array}{r}\text { tie } \\
\text { switches }\end{array}$ & $\begin{array}{c}\text { remotely controlled } \\
\text { switches }\end{array}$ & feeders & DG \\
units
\end{tabular}

methodology while ensuring reproducibility of our results, we describe in the next subsections the additional assumptions made regarding the location and characteristics of hosted DG, lines thermal limits, and number of automated switches.

A summary of the characteristics of these test systems is given in Table 3.

\subsection{Simulation assumptions}

The MIQCP optimization model has been developed in GAMS version 23.9.3 [22] and is solved using the simple branch and bound (SBB) solver. All tests have been performed on a PC of 2.8-GHz and 4-Gb RAM.

In each system we assume that all tie-switches and few sectionalizing switches are remotely controlled.

We consider four simulation cases differing in the type of control variables and values of thermal limits as follows:

- Case A: one removes overload only by DG units curtailment, assuming that all DG units are non-firm and hence eligible for curtailment; 
- Case B: one allows additionally manoeuvers on remote switches;

- Case C: compared to case B, one limits drastically the thermal ratings of tie lines, to further assess the optimization engines performances;

- Case D: compared to case B, one limits the number of remote switching actions in (22) to $\Delta S_{s w}=2$ (i.e. an open/close switching pair).

We consider voltage limits of 0.95 p.u. and 1.05 p.u. at all nodes. However, in our simulations we did not notice any voltage violation in the initial state or binding voltage limits at the optimal solution. Conversely, overload is always present in the initial state and sometimes thermal constraints are binding at the optimum (e.g. if reconfiguration does not suffice to remove overload).

\subsection{Results on the 33-bus distribution grid}

Fig. 4 shows the one-line diagram of the 33-bus network [21] which has been modified so as to host 6 identical DG units (G1 to G6) with the following characteristics: $P_{g}^{0}=0.6 \mathrm{MW}, Q_{g}^{0}=0 \mathrm{MVAr}, P_{g}^{\min }=0.1 \mathrm{MW}, P_{g}^{\max }=$ $0.6 \mathrm{MW}, Q_{g}^{\min }=-0.2 \mathrm{MVAr}$, and $Q_{g}^{\max }=0.1 \mathrm{MVAr}$. The active and reactive powers injected into the distribution system at node 0 are 0.188 MW and 1.754 MVar.

The set of remotely controlled switches comprises the 5 tie-switches (s33, s34, s35, s36, s37) and 5 well located sectionalizing switches (s8, s10, s20, s23, s27).

We set the thermal limit of all lines to 2 MVA, except of line 0-1 which has a limit of 10 MVA and line 4-5 which has a limit of 1 MVA. Given these 


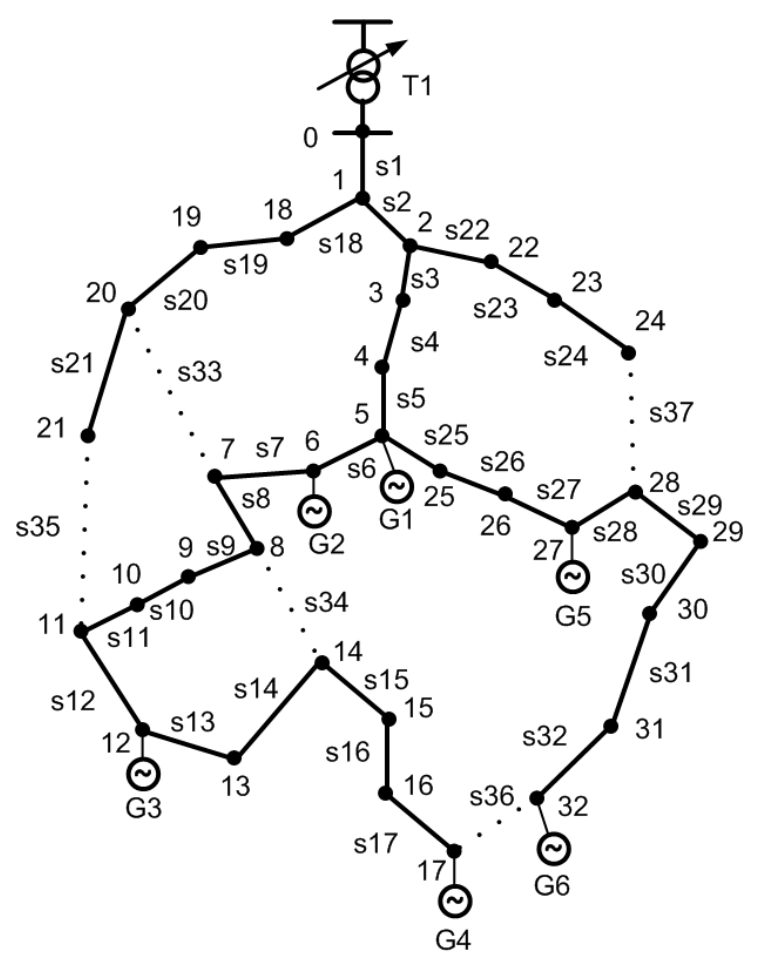

Figure 4: Modified 33-bus distribution grid 4

limits the line 4-5 gets overloaded due to the significant amount of feed-in, its limit being exceeded with 0.76 MVA.

\subsubsection{Effect of various objective functions and generators selection criterion}

We illustrate in case A the impact of the choice of the objective and the selection of generators and report the results in Table 4. According to the proposed DG selection criterion described in subsection 2.4 only generator G6 (see Fig. 4) does not induce consistent power flows in the sense of the overload. Therefore, the proposed approach in case "selected" does not curtail G6. When all generators participate in optimization (case "all") G6 is curtailed 
Table 4: 33-bus grid: non-firm DG units curtailment (MW)

\begin{tabular}{|c|c|c|c|c|c|}
\hline gen & $\begin{array}{c}L_{1} \\
\text { all }\end{array}$ & $\begin{array}{c}L_{2} \\
\text { all }\end{array}$ & $\begin{array}{c}L_{1} \\
\text { selected }\end{array}$ & $\begin{array}{c}L_{2} \\
\text { selected }\end{array}$ & $\begin{array}{c}L_{1 w} \\
\text { selected }\end{array}$ \\
\hline G1 & -0.5 & -0.088 & -0.5 & -0.106 & -0.214 \\
\hline G2 & -0.014 & -0.088 & -0.014 & -0.106 & -0.122 \\
\hline G3 & 0.0 & -0.086 & 0.0 & -0.103 & -0.092 \\
\hline G4 & 0.0 & -0.084 & 0.0 & -0.102 & -0.062 \\
\hline G5 & 0.0 & -0.088 & 0.0 & -0.105 & -0.028 \\
\hline G6 & 0.0 & -0.088 & - & - & - \\
\hline Overall & 0.514 & 0.522 & 0.514 & 0.522 & 0.518 \\
\hline
\end{tabular}

for the $L_{2}$ objective.

Regarding the objectives comparison, these results confirm the conclusions drawn for the example in Section 2, i.e. $L_{2}$ and $L_{1 w}$ objectives slightly increase the overall amount of DG curtailment compared to norm $L_{1}$.

\subsubsection{Further illustration of the approach}

In all simulations hereafter we use the settings $w_{g}=1$ and $w_{s}=0.01$ in the linear objective (4) for the sake of both illustrating cases where DG units are shut-down and provide reproducible benchmark test comparisons.

Table 5 presents numerical results with the approach in three cases. In case A the optimal DG units curtailment, which creates a counter-flow in line 4-5 and removes overload, consists of reducing the output of G1 to its minimum value and slightly reducing the output of $\mathrm{G} 2$. In cases B and D one can observe that by closing the tie switch s35 and opening the sectionalizing switch s10 the power from two units (G3 and G4) is rerouted and overload 
Table 5: 33-bus grid: DG units curtailment (MW) and switching actions

\begin{tabular}{|c|c|c|c|c|}
\hline Controls & Case A & Case B & Case C & Case D \\
\hline G1 & -0.5 & 0.0 & 0.0 & 0.0 \\
\hline G2 & -0.014 & 0.0 & 0.0 & 0.0 \\
\hline G3 & 0.0 & 0.0 & -0.237 & 0.0 \\
\hline Switches & & $\mathrm{s} 10, \mathrm{~s} 35$ & $\mathrm{~s} 8, \mathrm{~s} 35$ & $\mathrm{~s} 10, \mathrm{~s} 35$ \\
\hline Objective (p.u.) & 0.514 & 0.02 & 0.257 & 0.02 \\
\hline Time (s) & 0.21 & 1.35 & 1.57 & 1.12 \\
\hline
\end{tabular}

disappears without curtailing any DG, which proves the benefit of our approach. In case $\mathrm{C}$ the thermal limits of the 5 tie-lines have been decreased to 0.3 MVA. Due to this strong limitation the overload cannot be removed only by switching actions, and some generation is still curtailed, however to a less extent than in the case without switching.

We conclude that the additional degrees of freedom provided by remotely controlled network switches leads to a less DG curtailment which proves the advantage of the approach.

\subsection{Results on the 61-bus CREOS distribution network}

The network model was provided by the Luxembourgish grid operator (CREOS). We consider a virtual operating scenario where 20 DG units of various sizes are connected to the grid. We assume that due to the significant power feed-in one line in the first main feeder gets congested.

Table 6 presents the control actions to remove congestion in four cases. In case A the optimal DG curtailment consists of shutting-down G1, and reducing the output of $\mathrm{G} 2$ and G3. In cases $\mathrm{B}$ and $\mathrm{D}$, the congestion is 
Table 6: 61-bus grid: DG units curtailment (MW) and switching actions to remove congestion

\begin{tabular}{|c|c|c|c|c|}
\hline Controls & Case A & Case B & Case C & Case D \\
\hline \hline G1 & $-1.18^{*}$ & 0.0 & -0.86 & 0.0 \\
\hline G2 & -0.94 & 0.0 & 0.0 & 0.0 \\
\hline G3 & -0.58 & 0.0 & 0.0 & 0.0 \\
\hline G5 & 0.0 & 0.0 & -0.31 & 0.0 \\
\hline G6 & 0.0 & 0.0 & -0.53 & 0.0 \\
\hline Switches & & $\mathrm{s} 1, \mathrm{~s} 62$ & $\mathrm{~s} 17, \mathrm{~s} 63$ & $\mathrm{~s} 1, \mathrm{~s} 62$ \\
\hline Objective (p.u.) & 2.7 & 0.02 & 1.72 & 0.02 \\
\hline Time (s) & 0.43 & 3.3 & 24.4 & 3.11 \\
\hline
\end{tabular}

removed only by switching maneuvers, as by opening the sectionalizing switch s1 and closing the tie switch s62 the power from G3 and G4 is rerouted and congestion disappears without curtailing any DG. In case C, in order to illustrate how the method works when actions on both switches and DG plants are required to relief congestion, the thermal limits of the 3 tie-lines have been decreased to 1.0 MVA. Due to this strong limitation the congestion cannot be removed only by switching actions, and some generation is still curtailed, however to a less extent than in the case without switching.

\subsection{Results on the 84-bus distribution network}

In the 84-bus system we consider 15 identical DG units located at the following nodes (node 0 corresponds to the substation): 2, 3, 4, 5, 6, 7, 8, 9, 10, 20, 35, 40, 50, 72, 81 and 82. We assume that each DG produces initially $1 \mathrm{MW}$ and $0.3 \mathrm{MVar}$ and has the following limits $P_{g}^{\min }=0.1 \mathrm{MW}$, 
$P_{g}^{\max }=1 \mathrm{MW}, Q_{g}^{\min }=-0.2 \mathrm{MVAr}$, and $Q_{g}^{\max }=0.3 \mathrm{MVAr}$.

We assume that the line 2-3 gets overloaded (the thermal limit was set to $3 \mathrm{MVA}$ ). In case A generators at node 3 and 4 are curtailed with $0.9 \mathrm{MW}$ and 0.769 MW respectively. In case B no generator is curtailed as by opening the switch s4 and closing the tie switch s85 allows rerouting DG injections through another feeder. In case $\mathrm{C}$, where the thermal limit of all tie-switches has been decreased to 1 MVA, only switching actions are insufficient to relief congestion. The computational effort of these simulations is given in Table 7 .

\subsection{Results on the 136-bus distribution network}

In the 136-bus system we consider that 14 identical DG units, having the same characteristics as for the 84-bus system, are located at the following nodes (node 0 corresponds to the substation): 3, 6, 9, 12, 15, 25, 30, 45, 50, $60,90,100,115,120$, and 130 .

We assume that the line 2-3 gets overloaded (the thermal limit was set to 1 MVA). In case A generators at node 3 and 6 are curtailed with $0.9 \mathrm{MW}$ and $0.555 \mathrm{MW}$ respectively. In case B no generator is curtailed as by opening the switch s64 and closing the tie switch s136 the DG units located downstream this feeder are transferred to another feeder. In case $\mathrm{C}$, where the thermal limit of all tie-switches has been decreased to 1 MVA, only switching actions are insufficient to relief congestion. The CPU times of these computations are given in Table 7 .

\subsection{Scaling with system size}

Table 7 provides the computational times obtained in various experiments on the four test systems. 
Table 7: Summary of computational times (s)

\begin{tabular}{|c|c|c|c|c|}
\hline grid & Case A & Case B & Case C & Case D \\
\hline 33-bus & 0.21 & 1.35 & 1.57 & 1.27 \\
\hline 61-bus & 0.43 & 3.3 & 24.4 & 3.11 \\
\hline 83-bus & 0.55 & 6.3 & 9.5 & 6.0 \\
\hline 135-bus & 0.60 & 31.2 & 24.3 & 4.7 \\
\hline
\end{tabular}

One can remark that generally as long as both types of control actions (i.e. switches and DG output) are not interacting together (as in cases A and B) the optimal solution is obtained acceptably fast. On the other hand, as expected, the case $\mathrm{C}$ takes a bit longer time due to the strong interaction between both types of controls and the very tight constraints assumed. However, the program can be stopped after a desired elapsed time limit or as a certain optimality gap is reached and still provide a better solution than that obtained only by DG output curtailment. As expected the times in case $\mathrm{D}$ are smaller than in case $\mathrm{B}$ as the combinatorial space is further reduced by limiting the number of remote switches maneuvered. We conclude that these CPU times fulfill the on-line application requirements.

\section{Conclusions and future works}

This paper has contributed to various aspects of centralized, event triggered, optimization approaches for thermal constraint management in active radial distribution systems and has proved the interest and feasibility of the approach on a snapshot-basis for four benchmark and real world distribution systems. A salient feature of the scheme is that it optimizes jointly of DG 
units output and remotely controlled grid switches.

Results show that, for a given operation scenario, the use of remotely switching devices can lead in some cases to a significant reduction of curtailed generation, this option appearing thereby as an effective means to host larger amounts of DG in distribution grids. We have also illustrated the potential benefits of the approach in terms of energy savings using a simple 5-bus, 3-switches distribution system on a 24 hours basis. However, due to the very large combinatorial space stemming from the growing number of binary variables, applying the approach for more realistic test networks by incorporating many scenarios is very computationally challenging. As a consequence, future work is foreseen to thoroughly assess the approach in terms of energy savings in the context of pseudo real-time environment by using time-series $[4,6,7]$. We also plan to conduct further research to find more tractable problem approximations to relieve this computational burden.

The obtained computational times comply with real-time application requirements. Further speed-up is expected if the viability of switching actions is assessed by DSO at (operational) planning stage, ending up with a practical limited set of potential switching pairs to be checked on-line as overload occurs. This can also simplify the optimization model allowing problem decomposition and further enabling parallel processing.

The approach allows assessing the gain in terms of DG penetration thanks to the use of remotely controlled switches. It further can be used as a tool for trading-off the number of additionally deployed remotely controlled switches and the expected benefits, at least in terms of DG curtailment reduction.

Future work is also planned to extend the optimization model by imposing 
Table 8: Lines parameters: resistance, reactance and thermal limit

\begin{tabular}{|c|c|c|c|}
\hline line & $R(\Omega)$ & $X(\Omega)$ & $S_{\text {nom }}(\mathrm{MVA})$ \\
\hline \hline $0-1$ & 0.125 & 0.193 & 6 \\
\hline $0-2$ & 0.224 & 0.581 & 6 \\
\hline $1-2$ & 0.252 & 0.336 & 12 \\
\hline $1-3$ & 0.038 & 0.051 & 12 \\
\hline $2-4$ & 0.023 & 0.030 & 12 \\
\hline
\end{tabular}

additional constraints on fault current levels [25] so as to preserve the feeders' protection selectivity when transferring DG units from one feeder to another.

\section{Appendix}

Data of the 5-bus system

Table 8 provides the lines parameters for the 5 -bus $20 \mathrm{kV}$ test system of Fig. 1 and Table 9 yields the generators and load profiles for 24 hours. Load reactive power is assumed to be $10 \%$ of the load active power in all scenarios. We also assume two fixed generators at buses 3 and 4, not shown in the figure, which produce in all scenarios constant power of $1 \mathrm{MW}$ and $3 \mathrm{MW}$, respectively. For simplicity we assume that all generators keep their voltage at 1.0 p.u..

\section{Data of the 8-bus system}

Table 10 provides the data relative to the 8 -bus $20 \mathrm{kV}$ test distribution system of Fig. 3. All the 7 lines are identical having the resistance and reactance equal to $0.1 \Omega$ and $0.2 \Omega$, respectively, and the thermal limit of 3.5 
Table 9: Hourly generators and loads active power (MW)

\begin{tabular}{|c|c|c|c|c|c|c|c|c|c|c|c|c|}
\hline gen/load & 1 & 2 & 3 & 4 & 5 & 6 & 7 & 8 & 9 & 10 & 11 & 12 \\
\hline G1 & 0.09 & 0.09 & 0.09 & 0.09 & 0.11 & 0.28 & 0.96 & 2.27 & 4.10 & 5.74 & 6.90 & 7.53 \\
\hline G2 & 5.56 & 6.42 & 7.47 & 8.00 & 6.72 & 5.30 & 4.99 & 5.44 & 3.38 & 3.02 & 2.83 & 2.18 \\
\hline L1 & 3.39 & 3.26 & 3.28 & 3.26 & 3.36 & 3.55 & 4.14 & 4.21 & 5.00 & 3.31 & 1.79 & 1.35 \\
\hline L2 & 1.39 & 1.26 & 1.28 & 1.76 & 1.86 & 2.55 & 3.14 & 4.21 & 5.00 & 4.81 & 4.79 & 4.85 \\
\hline \hline gen/load & 13 & 14 & 15 & 16 & 17 & 18 & 19 & 20 & 21 & 22 & 23 & 24 \\
\hline \hline G1 & 7.87 & 8.00 & 7.74 & 6.83 & 5.69 & 4.13 & 2.44 & 1.05 & 0.34 & 0.10 & 0.09 & 0.09 \\
\hline G2 & 1.35 & 0.32 & 0.00 & 0.53 & 1.55 & 2.94 & 2.90 & 3.15 & 4.25 & 4.92 & 5.15 & 5.30 \\
\hline L1 & 0.96 & 0.69 & 0.57 & 0.59 & 0.79 & 1.26 & 1.89 & 2.90 & 4.34 & 4.41 & 4.41 & 4.31 \\
\hline L2 & 4.71 & 4.69 & 4.57 & 4.59 & 4.54 & 4.76 & 4.89 & 4.40 & 4.34 & 4.41 & 4.41 & 4.31 \\
\hline
\end{tabular}

MVA. For simplicity we assume that all generators keep their voltage at 1.0 p.u.. The active and reactive powers injected into the distribution system at node A are -3.695 MW and 1.853 MVar.

\section{Acknowledgements}

This work is conducted as part of the "Reliable and Efficient Distributed Electricity Generation in Smart Grids" (REDESG) project within the "CREOSSnT Framework Program" The authors are supported by the Fonds National de la Recherche, Luxembourg (C11/SR/1278568).

\section{References}

[1] A. Keane, L.F. Ochoa, C.L.T. Borges, G.W. Ault, A. D. AlarconRodriguez, R.A.F. Currie, F. Pilo, C. Dent, G.P. Harrison, State-of- 
Table 10: Generators and loads initial active/reactive powers (MW/MVar) and generators physical limits

\begin{tabular}{|c|c|c|c|c|c|c|}
\hline gen/load & $P_{0}$ & $Q_{0}$ & $P_{g}^{\min }$ & $P_{g}^{\max }$ & $Q_{g}^{\min }$ & $Q_{g}^{\max }$ \\
\hline \hline G1 & 3.0 & -1.494 & 0.1 & 3.0 & -2.0 & 2.0 \\
\hline G2 & 1.0 & -0.30 & 0.1 & 1.0 & -1.0 & 1.0 \\
\hline G3 & 1.5 & 0.90 & 0.1 & 1.5 & -1.0 & 1.0 \\
\hline G4 & 1.2 & 0.05 & 0.1 & 1.2 & -1.0 & 1.0 \\
\hline L2 & 0.2 & 0.1 & & & & \\
\hline L3 & 0.7 & 0.3 & & & & \\
\hline L4 & 2.1 & 0.6 & & & & \\
\hline
\end{tabular}

the-Art Techniques and Challenges Ahead for Distributed Generation Planning and Optimization, IEEE Trans. Power Syst. 28 (2) (2013) $1493-1502$.

[2] P.S. Georgilakis, N.D. Hatziargyriou, Optimal Distributed Generation Placement in Power Distribution Networks: Models, Methods, and Future Research, IEEE Trans. Power Syst. 28 (3) (2013) 3420-3428.

[3] R.A.F. Currie, C.E.T. Foote, G.W. Ault, J.R. McDonald, Active power flow management utilising operating margins for the increased connection of distributed generation, IET Generation, Transmission, Distribution 1 (1) (2007) 197-202.

[4] M.J. Dolan, E.M. Davidson, I. Kockar, G.W. Ault, S.D.J. McArthur, Distribution power flow management utilizing an online optimal power flow technique, IEEE Trans. Power Syst. 27 (2) (2012) 790-799. 
[5] M.J. Dolan, E.M. Davidson, G.W. Ault, K.R.W. Bell, S.D.J. McArthur, Distribution Power Flow Management Utilizing an Online Constraint Programming Method, IEEE Trans. Smart Grid 4 (2) (2013) 798-805.

[6] T. Boehme, G.P. Harrison, A.R. Wallace, Assessment of distribution network limits for non-firm connection of renewable generation, IET Renewable Power Generation 4 (1) (2010) 64-74.

[7] T. Sansawatt, L.F. Ochoa, G.P. Harrison, Smart decentralized control of DG for voltage and thermal constraint management, IEEE Trans. Power Syst. 27 (3) (2012) 1637-1645.

[8] F. Capitanescu, J.L. Martinez Ramos, P. Panciatici, D. Kirschen, A. Marano Marcolini, L. Platbrood, L. Wehenkel, Security-constrained optimal power flow: state-of-the-art, challenges, and future trends, Elec. Pow. Syst. Research, 81 (8) (2011) 1731-1741.

[9] R.D. Christie, B.F. Wollenberg, I. Wangensteen, Transmission management in the deregulated environment, Proc. of the IEEE 88 (2) (2000) 170-195.

[10] Per Hallberg (Chair) et al., Active Distribution System Management a key tool for the smooth integration of distributed generation, Eurelectric TF Active System Management, 2013, available on-line.

[11] F. Pilo, G. Pisano, G. Soma, Optimal Coordination of Energy Resources With a Two-Stage Online Active Management, IEEE Trans. on Industrial Electronics 58 (10) (2011) 4526-4537. 
[12] M. Lavorato, J.F. Franco, M.J. Rider, R. Romero, Imposing Radiality Constraints in Distribution System Optimization Problems, IEEE Trans. on Power Syst. 27 (1) (2012) 172-180.

[13] E. Romero Ramos, J. Riquelme Santos, J. Reyes, A simpler and exact mathematical model for the computation of the minimal power losses tree, Elec. Pow. Syst. Research 80 (5) (2010) 562-571.

[14] I. Bilibin, F. Capitanescu, J. Sachau, Overloads Management in Active Radial Distribution Systems: an Optimization Approach Including Network Switching, IEEE PES Powertech conference, Grenoble (France), June 2013.

[15] J.M. Lopez-Lezama, A. Padilha-Feltrin, J. Contreras, J.I. Munoz, Optimal Contract Pricing of Distributed Generation in Distribution Networks, IEEE Trans. on Power Syst. 26 (1) (2011) 128-136.

[16] P. Siano, L.F. Ochoa, G.P. Harrison, A. Picollo, Assessing the strategic benefits of distributed generation ownership for DNOs, IET Generation, Transmission, Distribution 3 (3) (2009) 225-236.

[17] D. Kirschen, R. Allan, G. Strbac, Contributions of Individual Generators to Loads and Flows, IEEE Trans. on Power Syst. 12 (1) (1997) 52-60.

[18] J. Bialek, Tracing the flow of electricity, IEE Gen., Transm. and Distrib. 143 (4) (1996) 313-320.

[19] A. Gomez Exposito, E. Romero Ramos, Reliable load flow technique for radial distribution networks, IEEE Trans. on Power Syst. 14 (3) (1999) 1063-1069. 
[20] R. Singh, B.C. Pal, R.A. Jabr, R.B. Vinter, Meter placement for distribution system state estimation: an ordinal optimization approach, IEEE Trans. on Power Syst. 26 (4) (2011) 2328-2335.

[21] M.E. Baran, F.F. Wu, Network Reconfiguration in Distribution Systems for Loss Reduction and Load Balancing, IEEE Trans. Power Delivery 4 (2) (1989) 1401-1497.

[22] B.A. McCarl, GAMS User Guide, Version 23.8, 2012. Available online: www.gams.com.

[23] C.T. Su, C.S. Lee, Network reconfiguration of distribution systems using improved mixed-integer hybrid differential evolution, IEEE Trans. on Power Delivery 18 (3) (2003) 1022-1027.

[24] J.R.S. Mantovani, F. Casari, R. A. Romero, Reconfiguracao de sistemas de distribuicao radiais utilizando o criterio de queda de tensao, SBA Controle \& Automacao 11 (3) (2000) 150-159.

[25] P. Vovos, J. Bialek, Direct incorporation of fault level constraints in optimal power flow as a tool for network capacity analysis, IEEE Trans. on Power Syst. 20 (4) (2005) 2125-2134. 